TAUP-2342-96

hep-ph/9606284

June 1996

\title{
Passage of charmed particles through the mixed phase in high-energy heavy-ion collisions
}

\author{
Benjamin Svetitsky円 \\ Asher Uziel[] \\ School of Physics and Astronomy \\ Raymond and Beverly Sackler Faculty \\ of Exact Sciences \\ Tel Aviv University \\ $69978 \mathrm{Tel}$ Aviv, Israel
}

PACS numbers: 12.38.Mh, 12.38.Qk, 14.40.Lb, 52.25.Fi

\begin{abstract}
$A B S T R A C T$. We employ a modified cascade hydrodynamics code to simulate the phase transition of an expanding quark-gluon plasma and the passage of a charmed particle through it. When inside the plasma droplets, the charmed quark experiences drag and diffusion forces. When outside the plasma, the quark travels as a $D$ meson and experiences collisions with pions. Additional energy transfer takes place when the quark enters or leaves a droplet. We find that the transverse momentum of $D$ mesons provides a rough thermometer of the phase transition.
\end{abstract}

\footnotetext{
${ }^{1}$ E-mail: bqs@julian.tau.ac.il

${ }^{2}$ Current address: Scitex Corporation, P.O.B. 330, 46103 Herzlia B, Israel
} 


\section{Introduction}

Charmed particles are promising candidates for probing the early stages of ultrarelativistic heavy-ion collisions. Because of their large mass, they can be created only in the initial hard collisions between the nuclear constituents [1]. Most charmed quarks created in hadronic collisions emerge with nonrelativistic velocities [2], and hence in nuclear collisions they will remain in the collision region for a long time. Finally, the passage of charmed quarks through the plasma is a problem amenable to theoretical analysis [3, 4, 5, 6]. Thus the observation of charmed particles, and in particular the measurement of their transverse momentum distribution, should yield information about the hot environment present early in the system's evolution.

Consider a high-energy nuclear collision which leads to creation in the central rapidity region of both a charmed quark pair and a quark-gluon plasma. We adopt Bjorken's scaling hydrodynamics ansatz to describe the plasma [0, 8]. The initial creation of the $c \bar{c}$ pair takes place in a time on the order of $1 / m_{c} \simeq 0.1 \mathrm{fm} / c$. Within a few tenths of $\mathrm{fm} / c$ the quarks will find themselves in an expanding quark-gluon plasma near local equilibrium [9]. We begin following the progress of each quark at this time, $\tau=\tau_{0}$, when the temperature is $T_{0}$. At this point, we assume, each quark begins a process of scattering and diffusion in the plasma which causes a loss of initial momentum and relaxation towards the (changing) thermal velocity.

The plasma expands and cools according to $T=T_{0} \cdot\left(\tau / \tau_{0}\right)^{-1 / 3}$, until at $\tau=\tau_{p}$ the phase transition begins, the temperature having reached $T=T^{*}$, the transition temperature. The "mixed phase" forms as follows. If there is a genuine first-order transition, the plasma supercools slightly, and then bubbles of the low-temperature phase are nucleated by fluctuations or by inhomogeneities. These bubbles grow, meet, and coalesce, and eventually we have a hadron gas containing mostly pions and also droplets of plasma which are evaporating. The phase transition is complete when the last of the droplets evaporate into hadrons, at $\tau=\tau_{h}$. The enormous entropy of the plasma which must be converted to hadrons dictates that $\tau_{h}$ is more than ten times $\tau_{p}$ : The mixed phase is the longest stage in the evolution of the system.

At the beginning of the mixed phase each charmed quark is in plasma, but it may emerge into a region of hadron gas, and then it might be reabsorbed into plasma only to emerge again [10. Emergence into the hadron 
gas requires hadronization, the binding of the charmed quark with a light antiquark to form a meson; reabsorption involves stripping off the antiquark by collisions with the plasma. We will discuss the processes of hadronization and ionization in detail below; suffice it for now to observe that hadronization involves a loss of kinetic energy by the quark, enough to supply at least the difference $m_{D}-m_{c} \simeq 350 \mathrm{MeV}$. If the quark does not have this kinetic energy to lose, it may be trapped in the plasma. It could emerge later if it gains enough energy through diffusion; otherwise it is trapped in the plasma until the plasma finally evaporates. Of course, it must hadronize at least once. Whenever the quark/meson is in a region of hadron gas, it is liable to scatter off the hadrons, mostly pions, and thus to undergo yet more drag and diffusion.

The situation is not too different if there is no real phase transition. The high- and low-temperature regimes are still distinguished by very different entropy densities. The "mixed phase" in this case would be a mixture of regions with temperatures slightly above and slightly below the crossover temperature at which the entropy changes steeply. The notion of boundaries between droplets of the pure phases should still be valid, as should the estimates of $\tau_{p}$ and $\tau_{h}$.

For simulation of the mixed phase we adopt the cascade hydrodynamics model [11] of Bertsch et al. In this model, when the temperature reaches $T^{*}$ the plasma simply breaks up into droplets. As the system continues its longitudinal expansion the droplets become ever more rarefied, all the while radiating and reabsorbing pions. Finally the droplets all evaporate, completing the phase transition. We have supplemented the cascade code with the routines needed to follow the progress of a charmed quark/meson through the system.

In the next section we describe the various assumptions of our model and how they are implemented in the simulation program [12]. Proceeding step-by-step through the collision process, we discuss the initial conditions given the plasma and the charmed quark diffusing therein; the Langevin process by which this diffusion takes place; and the cascade hydrodynamics by which the plasma droplets and pions are simulated in the mixed phase? We then present the model which is central to the physics presented here, that of charm hadronization and re-ionization [10], and finally discuss the

\footnotetext{
${ }^{3}$ This is essentially unchanged from the algorithm originally used by Bertsch et al. 11]
} 
$D \pi$ scattering which occurs when the charmed quark is outside the plasma.

In Section 3 we present our results, which are mainly predictions for the final $p_{\perp}$ of a charmed meson born of a $c$ quark created with initial $p_{\perp}=p_{\perp 0}$. We plot this quantity for a range of assumptions concerning the free parameters of the simulation. Our main qualitative result may be simply stated: $D$ mesons do provide a rough thermometer of the phase transition. This is because a large percentage of the charmed quarks are trapped in the plasma until it breaks up; moreover, those which escape the plasma droplets early will experience many collisions in the pion gas which has not yet rarefied. Unfortunately, the temperature scale of the thermometer contains uncertainties due to the dynamical assumptions we make, chiefly the initial size of the plasma droplets. The measurement of temperature is also degraded by transverse flow of the droplets and pions.

\section{Ingredients of the simulation}

\subsection{Initial conditions}

The initial conditions we specify are of two classes. One describes the cylinder of plasma at proper time $\tau_{0}$, while the other gives the initial momentum and location of each charmed quark.

The plasma is assumed to occupy a cylinder of fixed cross section $R$, equal to the radius of the colliding nuclei. We consider only central $U U$ collisions, and hence $R \simeq 7 \mathrm{fm}$. The initial time $\tau_{0}$ is the time at which the plasma is taken to be fully formed. We can estimate it from the flux tube model [13] according to [14] $\tau_{0} \simeq(1 \mathrm{fm}) \cdot A^{-1 / 6} \simeq 0.5 \mathrm{fm}$.

We fix the initial temperature in the usual way [15] by setting the eventual pion multiplicity $d N_{\pi} / d y$. This is related to the entropy density at freezeout by $d S / d y=3.6 d N_{\pi} / d y$. The cascade simulation predicts an entropy increase of $20-30 \%$ during the phase transition [11]; if we take this into account, while neglecting the entropy created through dissipation at other stages of the expansion, then the entropy density $d S / d y$ at $\tau_{0}$ is $(1.2)^{-1}$ times that at freezeout. Setting

$$
\frac{d S}{d y}=\left(8+6 N_{f} \frac{7}{8}\right) \frac{4 \pi^{2}}{45} T^{3} \cdot \pi R^{2} \tau
$$


in the quark-gluon plasma, we determine the temperature $T_{0}$ at $\tau_{0}$. (For simplicity we take $N_{f}=2$.) We present results for an initial temperature of $300 \mathrm{MeV}$, corresponding to $d N / d y=1400$.

The hard collision of the incident nuclei takes place at $t=z=0$, and hence the charmed quarks are created on trajectories emanating from this surface and thus satisfying $z=v_{\ell} t$ for some $v_{\ell}$. We follow one quark at a time, and so the longitudinal-boost invariance of the fluid allows us to work in the frame where $v_{\ell}=0$. By choosing the $x$ axis along the quark's initial position we end up, with no loss of generality, with $\mathbf{x}_{0}=\left(x_{0}, 0,0\right)$ and $\mathbf{p}_{0}=\left(p_{0}^{x}, p_{0}^{y}, 0\right)$.

The radius $x_{0}$ at which the heavy quark is created is taken from a distribution proportional to the number of nucleon-nucleon collisions taking place at that radius,

$$
p\left(x_{0}\right) d x_{0} \propto\left(R^{2}-x_{0}^{2}\right) 2 \pi x_{0} d x_{0} .
$$

Azimuthal symmetry of the parton-parton collision gives the direction of $\mathbf{p}_{0}$ in the $x y$ plane a uniform distribution. The magnitude $p_{0}$ is an input parameter.

Charmed quarks are of course created in pairs. We neglect entirely the interaction between the quark and antiquark, and any influence they may have on each other as they diffuse through the plasma and mixed phases. The reason is that the two are created with opposite transverse momenta (and different rapidities) which carry them apart quickly. Since $\tau_{p}$ is (under our assumptions) at least $1.7 \mathrm{fm} / c$, the quarks can be $3 \mathrm{fm}$ apart by the onset of the phase transition; since the droplets in the mixed phase have initial radii of 1-2 fm (see below), the quark and antiquark will generally find themselves in different droplets. Their interaction will therefore be indirect at best. We have not studied correlations in the directions of the produced $D$ pair [16], which may offer another diagnostic probe of the phase transition.

\footnotetext{
${ }^{4}$ Actually for reasons of economy we put two charmed quarks into each event. We make sure that they have no effect on each other by giving them different initial values of $x_{0}$, with opposite sign, and the same $\mathbf{p}_{0}$.

${ }^{5}$ The rare case where the $c \bar{c}$ pair are found in the same droplet might make a significant contribution to $J / \psi$ production.
} 


\subsection{Diffusion before the phase transition}

From $\tau=m_{c}^{-1}$ to $\tau=\tau_{0}$ the interaction region is in a rapidly changing state of particle creation and equilibration. Since this period is very short, it doesn't make much difference how we model it. For simplicity, we proceed as if it were an equilibrium plasma at $T=T_{0}$. We allow the charmed quark to diffuse in this plasma in the same way as it does after $\tau_{0}$, which we now describe.

From $\tau_{0}$ to $\tau_{p}$ the charmed quark is in a pure plasma. We model its diffusion by a non-relativistic Langevin equation,

$$
\frac{d \mathbf{p}}{d t}=-\gamma(T) \mathbf{p}+\boldsymbol{\eta},
$$

where $\boldsymbol{\eta}$ is a Gaussian noise variable, normalized such that

$$
\left\langle\eta_{i}(t) \eta_{j}\left(t^{\prime}\right)\right\rangle=\alpha(T) \delta_{i j} \delta\left(t-t^{\prime}\right) .
$$

Both the drag coefficient $\gamma$ and the momentum-space diffusion coefficient $\alpha$ depend on the local temperature. We take $\gamma$ from the result of [4], which we parameterize as

$$
\gamma(T)=a T^{2}, \quad a=2 \times 10^{-6} \mathrm{fm}^{-1} \mathrm{MeV}^{-2} .
$$

(We neglect any momentum dependence in $\gamma$.) $\gamma$ and $\alpha$ are related by the fluctuation-dissipation relation, which says that in equilibrium

$$
\left\langle p_{i}^{2}\right\rangle=\frac{\alpha}{2 \gamma} .
$$

For temperatures where the quark in equilibrium is non-relativistic, we would have $\left\langle p_{i}^{2}\right\rangle=m_{c} T$; in the range $T=150-250 \mathrm{MeV}$, however, $\left\langle p_{i}^{2}\right\rangle$ is up to $33 \%$ larger than this. As a relativistic correction, we calculate $\alpha$ from (6) with $\left\langle p_{i}^{2}\right\rangle=1.33 m_{c} T$.

We apply (3), which is not Lorentz-invariant, in the rest frame of the plasma surrounding the quark. In the Bjorken scaling expansion we assume, this is a frame moving longitudinally with $v_{z}=z / t$, where $z$ and $t$ are the coordinates of the quark. The local temperature is given by $T=T_{0}\left(\tau_{0} / \tau\right)^{1 / 3}$.

\footnotetext{
${ }^{6}$ We here correct an error in $[10]$.
} 


\subsection{Simulation of the mixed phase}

The cascade hydrodynamics model [11] of the mixed phase assumes that at proper time $\tau_{p}$, the beginning of the phase transition, the plasma breaks up into droplets. These droplets subsequently emit and absorb pions. The droplets' interior is always at the transition temperature $T^{*}$ and thus their energy density is fixed at

$$
\varepsilon=\left(8+6 N_{f} \frac{7}{8}\right) \frac{\pi^{2}}{15} T^{* 4}+B
$$

where $B$ is the bag constant. We worked with two values for the transition temperature, $T^{*}=150 \mathrm{MeV}$ and $T^{*}=200 \mathrm{MeV}$, with $B$ adjusted accordingly. Table 1 shows the values of various quantities for the two cases.

A droplet with radius $r_{d}$ has mass equal to

$$
M_{d}=\frac{4}{3} \pi r_{d}^{3} \varepsilon
$$

The initial value of $r_{d}$ determines how long it takes the droplets to evaporate. If the transition were adiabatic, the lifetime of the mixed phase would be determined by entropy conservation, which makes the ratio of times equal to the ratio of entropy densities,

$$
\frac{\tau_{h}}{\tau_{p}}=\frac{\sigma_{p}}{\sigma_{h}} \simeq 10
$$

In the cascade model, however, the expansion is not adiabatic and the duration of the transition, that is, the time needed for the droplets to evaporate, is strongly dependent on the droplets' initial radius. Thus larger initial radii give a longer-lived mixed phase.[7 We experimented with different initial values for $r_{d}$ ranging from $1 \mathrm{fm}$ to $2 \mathrm{fm}$. As each droplet emits and absorbs pions its mass changes, and $r_{d}$ changes accordingly.

The program actually steps through the time coordinate $t$ rather than the proper time $\tau$. At each time $t$ we determine the $z$ coordinates of the two points on the hyperbola $\tau=\tau_{p}$, where $\tau \equiv \sqrt{z^{2}-t^{2}}$ : This is where the phase transition is currently occurring, and thus where droplet creation should take

\footnotetext{
${ }^{7}$ We find that for $r_{0}=1.0,1.5$, and 2.0 fm the duration of the phase transition turns out to be 12,22 , and $32 \mathrm{fm} / c$, respectively, independent of the transition temperature.
} 


\begin{tabular}{lcc|c|c} 
Transition temperature & $T^{*}$ & & $150 \mathrm{MeV}$ & $200 \mathrm{MeV}$ \\
\hline Beginning time of transition & $\tau_{p}$ & $(\mathrm{fm})$ & 4.0 & 1.7 \\
Bag constant & $B^{1 / 4}$ & $(\mathrm{MeV})$ & 208 & 278 \\
Plasma energy density & $\varepsilon$ & $\left(\mathrm{GeV} / \mathrm{fm}^{3}\right)$ & 1.0 & 3.2 \\
Thermal pion energy & $E_{\pi}^{T}$ & $(\mathrm{MeV})$ & 480 & 610 \\
Thermal pion momentum & $p_{\pi}^{T}$ & $(\mathrm{MeV})$ & 520 & 680 \\
Thermal $D$ momentum & $p_{D}^{T}$ & $(\mathrm{MeV})$ & 1010 & 1200
\end{tabular}

Table 1: Physical quantities for the two transition temperatures considered. $p_{\pi}^{T}$ and $p_{D}^{T}$ are root-mean-square momenta, $p^{T} \equiv \sqrt{\left\langle\mathbf{p}^{2}\right\rangle}$, while $E^{T} \equiv\langle E\rangle$.

place. The droplets are uniformly distributed across the cylinder, and their number in the $z$-slice at $\tau_{p}$ is fixed to conserve the entropy of the plasma at the breakup. A droplet's initial momentum is thermally distributed in the locally comoving rest frame, which has rapidity $y=\tanh ^{-1}(z / t)$.

From the time of its formation, each droplet interacts with the pion gas. By detailed balance, the pion emission rate is equal to the absorption rate in equilibrium [17]. The latter is determined by assuming that a droplet absorbs all pions that approach within a distance $d=1 \mathrm{fm}$ from its surface. The absorption rate in equilibrium is thus $1 / 4$ of the pion flux times the area of the extended droplet surface,

$$
W=\frac{1}{4} n_{\pi}^{\mathrm{eq}} v_{\pi} \cdot 4 \pi\left(r_{d}+d\right)^{2} .
$$

We take

$$
n_{\pi}^{\mathrm{eq}}=3 \frac{16}{\pi^{2}} \zeta(3) T^{3}
$$

and $v_{\pi}=1$, as appropriate for ultrarelativistic pions. Each emitted pion is created at a random place on the droplet's surface, with a thermally distributed momentum (in the droplet's rest frame) directed outward. The emission process conserves energy and momentum exactly through the recoil and shrinkage of the rigid droplet. Pion absorption is simpler, consisting of the disappearance of any pion which ventures within a distance $d$ of a droplet's surface (in its rest frame), with its four-momentum taken up by the droplet's recoil and growth.

If the droplet contains less rest energy than that required to make four thermal pions (see Table 1), it falls apart altogether into $n$ thermally dis- 
tributed pions. $n$ might in fact be less than four, because if $M_{d} / m_{\pi}<4$ then $n$ is given by the integer part of this ratio. As in [1], we adopt the simple model of first giving each pion a random, thermally-distributed momentum; the momenta are then shifted and rescaled to conserve the momentum and energy of the droplet.

$\pi \pi$ scattering is taken to be isotropic, with an isospin- and energy-dependent cross section of about $30 \mathrm{mb}$. Droplet-droplet scattering is neglected entirely, which is reasonable after the earliest times because dilution by the longitudinal expansion is rapid.

\subsection{Charm hadronization and re-ionization}

At the beginning of the phase transition the charmed quark is inside a droplet. (We restart the simulation if the random creation of droplets leaves the quark outside.) It continues to undergo diffusion in the plasma as it did before the transition time. The Langevin equation (3) is applied in the droplet's rest frame via the appropriate Lorentz transformation, and every change in the quark's momentum is balanced by recoil of the droplet (and a change in its mass to conserve energy). Sooner or later, the quark strikes the wall of the droplet.

Our model of hadronization is based on a picture of a quark incident on a static bag boundary [18, 19, 20]. Upon striking the wall the quark stretches a flux tube [19] outward which we assume to be always radially oriented. The tube possesses a tension (energy density) $\sigma=0.16 \mathrm{GeV}^{2}$ in the droplet rest frame, and we take this to be the only force acting on the quark during this period. Thus we integrate the equation

$$
\frac{d \mathbf{p}}{d t}=-\sigma \hat{\mathbf{r}}
$$

in the droplet frame until either the quark reenters the droplet or the flux tube breaks. 8

The flux tube fissions at a rate $d \Gamma / d \ell$ per unit length. Various estimates for this quantity are $0.3 \mathrm{fm}^{-2}$ from the flux tube model [19] and anywhere from 0.5 to $2.6 \mathrm{fm}^{-2}$ from comparisons of string fragmentation models with

\footnotetext{
${ }^{8}$ This is the weighted average at $T=200 \mathrm{MeV}$. See [11] for details.

${ }^{9}$ The droplet also reacts to the tension of the tube.
} 
experiment [21]. We adopt two values for $d \Gamma / d \ell$ at the extremes of the range, 0.5 and $2.5 \mathrm{fm}^{-2}$.

The tube breaks at a point $\mathcal{P}$ some distance $\ell$ from its base. The segment from $\mathcal{P}$ to the droplet is reabsorbed in the droplet; the remainder of the tube snaps back into the quark and turns it into a meson. The $D$ emerges with energy (in the droplet rest frame) $E_{D}=E_{c}+\sigma(L-\ell$ ), where $L$ is the total length of the tube. Clearly $\sigma(L-\ell)$ cannot be less than $m_{D}-E_{c}$, or there will not be enough energy for hadronization at all.

A quark which strikes the droplet wall with energy $E_{c}<m_{D}$ would be unable to hadronize if the droplet were static. Its reflection from the droplet wall, however, still involves stretching a flux tube and this process can take $1.5 \mathrm{fm} / c$ in the droplet rest frame. During this time, the droplet emits and absorbs pions and thus its recoil might stretch the flux tube further."Th This effect, which of course applies as well to situations with $E_{c}>m_{D}$, sometimes enables even quarks which are initially below threshold to hadronize. Again, the flux tube breaks at a random point, with the constraint $\sigma(L-\ell)>$ $m_{D}-E_{c}$ in the droplet's new rest frame.

When the tube breaks, the resulting $D$ meson emerges immediately with its momentum in the same direction as that of the quark. Four-momentum is conserved via recoil of the droplet. In the droplet's initial rest frame, the kinetic energy of recoil comes from the attached segment of the flux tube and from a change in its rest mass,

$$
M_{d}^{\prime 2}+(\Delta p)^{2}=\left(M_{d}+\sigma \ell\right)^{2}
$$

where $\Delta p \equiv p_{c}-p_{D}$ is the momentum transfer from the quark/meson.

It is possible for the $D$-meson to be created with momentum directed towards the droplet, and even for it to collide with the droplet in the next time step. At that point the quark is reabsorbed in an ordinary ionization process (see below), which means it has been effectively reflected back into the droplet.

We neglect entirely an additional possibility for hadronization, namely, that the $c$ quark might encounter a light antiquark as it nears the droplet

\footnotetext{
${ }^{10}$ We neglect the possibility of fragmentation of the quark into a $D^{*}$ or a $D \pi$ pair, because of the sharply reduced phase space for such fragmentation.

${ }^{11} \mathrm{~A}$ change in the droplet's radius also causes the flux tube to change its length, conserving energy via the droplet's mass.
} 
surface. It could thus hadronize without forming a flux tube, and possibly even gain energy in the process. We calculate the probability of this to be small, as follows. The density of light antiquarks at $T=200 \mathrm{MeV}$ is $n_{q}=1 \mathrm{fm}^{-3}$, whence the flux of outward-going antiquarks at the droplet surface is $j_{q}=n_{q} / 4$. An antiquark must appear within a range $R \simeq 0.5 \mathrm{fm}$ of the $c$ quark during the reflection process which takes $\Delta t \simeq 1.5 \mathrm{fm} / c$. The relative probability of forming a color singlet is $1 / 9$. Thus the probability of hadronization through light-quark binding is only

$$
P=j_{q} \cdot\left(\pi R^{2}\right) \cdot \Delta t \cdot \frac{1}{9} \simeq 3 \% \text {. }
$$

The only remaining possibility for hadronization is that the droplet breaks up around the $c$ quark, which happens when the mass of the droplet falls below the energy needed to make four thermal pions plus the energy needed for hadronization. In our scenario, the charmed quark picks up matter which was at rest in the droplet rest frame, and thus its three-momentum is conserved, $p_{D}=p_{c}$, while the energy required for creation of the $D$ is supplied by the droplet. Having given this energy to the meson, the droplet then breaks up according to the rule given above for ordinary droplet breakup.

If the $c$ quark is inside a flux tube at the time the droplet breaks up, the flux tube's energy is merely added to that of the droplet, and the remainder of the breakup is handled as above.

The inverse of hadronization, ionization, occurs when a $D$ meson strikes a droplet, and is easily dealt with. We assume that the light quark is simply stripped off the meson, leaving a $c$ quark with the same velocity $v_{c}=v_{D}$ (a frame-independent statement). This means that the quark/meson loses energy since $E_{c}=\left(m_{c} / m_{D}\right) E_{D}$. As noted in [10], a $D$ meson which undergoes ionization and re-hadronization in quick succession would lose energy in the amount

$$
\begin{aligned}
\Delta E & =\Delta E_{\text {ion }}+\Delta E_{\text {had }}=E_{D}\left(1-\frac{m_{c}}{m_{D}}\right)+0 \\
& \geq m_{D}\left(1-\frac{m_{c}}{m_{D}}\right) \simeq 370 \mathrm{MeV} .
\end{aligned}
$$

A quark with less kinetic energy than this will get trapped in the droplet. Of course, our simulation actually follows in detail the diffusion of the $c$ quark inside the droplet so the estimate (15) is only a rough guide. 


\subsection{Scattering in the hadron gas}

Whenever the charmed quark is outside a droplet it moves as a $D$ meson. It can then scatter off the pions which have been emitted from the droplets. We use a simple model of isotropic, energy-independent, and charge-independent scattering, with total cross-section $\sigma_{D \pi}=9 \mathrm{mb}$. We arrive at this number via the additive quark model, as follows. Beginning with the (high-energy) $p p$ cross section, $\sigma_{p p}=40 \mathrm{mb}$, we estimate that the cross section for light quark scattering is $\sigma_{q q}=\sigma_{p p} / 9 \simeq 4 \mathrm{mb}$. Similarly we begin with the $\psi N$ cross section [22], $\sigma_{\psi N}=2 \mathrm{mb}$, and estimate that the cross section for charmed quarks on light quarks is $\sigma_{c q} \simeq 0.3 \mathrm{mb} \ll \sigma_{q q}$. Thus we arrive at $\sigma_{D \pi}=$ $2 \sigma_{q q}+2 \sigma_{c q} \simeq 9 \mathrm{mb}$. We ignore resonant scattering via the $D^{*}$ since the resonances lie at $p=39-44 \mathrm{MeV} / c$ in the center-of-momentum frame, which is far below the typical thermal momenta (see Table 1).

(This procedure gives good results for $K \pi$ scattering. Here one begins with $\sigma_{\phi N}=13.8 \mathrm{mb}$ [23] and reaches $\sigma_{K \pi} \simeq 13 \mathrm{mb}$, which is not far from the values from phase shift analysis [24] $8 \mathrm{mb}<\sigma_{K \pi}<13 \mathrm{mb}$ in the energy range $1 \mathrm{GeV}<\sqrt{s}<1.5 \mathrm{GeV}$, above the $K^{*}$ resonance.)

\section{Numerical results}

The aim of our calculation is a prediction for the $p_{\perp}$ distribution of $D$ mesons created in heavy ion collisions. The result naturally depends on the distribution one assumes for the initial momentum of the $c$ quark. Rather than tie ourselves to specific model predictions for the latter [2], we present our results as plots of the RMS $p_{\perp}^{D}$ for given initial quark momentum $p_{\perp 0}^{c}$ in the range between $1 \mathrm{GeV}$ and $2 \mathrm{GeV}$.

Our main result is apparent in the last figure, which shows that $p_{\perp}^{D}$ can be a thermometer of the mixed phase. We begin, however, with a detailed analysis of the numerical results for $T^{*}=150 \mathrm{MeV}$. In Fig. 11 we show the calculated $p_{\perp}^{D}$ for two values of the initial droplet radius $r_{0}$ and of the flux-tube fission rate $d \Gamma / d \ell$, as discussed above. We note two features immediately: (1) $p_{\perp}^{D}$ varies but weakly with $p_{\perp 0}^{c}$, showing the effects of drag and thermalization; [ए] (2) $p_{\perp}^{D}$ lies generally above its thermal value $\sqrt{\frac{2}{3}} p_{D}^{T}=820 \mathrm{MeV}$ (see

\footnotetext{
${ }^{12}$ The dependence will be even weaker for $p_{\perp 0}^{c}<1 \mathrm{GeV}$ because thermalization will wipe out any memory of the initial momentum.
} 


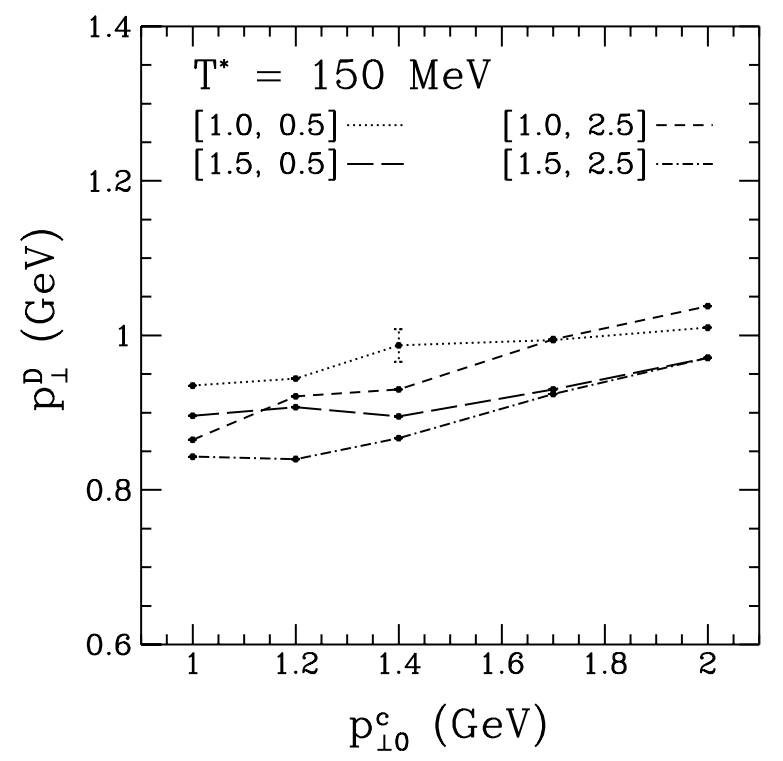

Figure 1: RMS transverse momentum of $D$ meson vs. initial transverse momentum of $c$ quark, for transition temperature $T^{*}=150 \mathrm{MeV}$. The four curves correspond to different values of $r_{0}$ and $d \Gamma / d \ell$ (in $\mathrm{fm}$ and $\mathrm{fm}^{-2}$, respectively): [1.0, 0.5] (dotted), [1.0, 2.5] (short dashes), [1.5, 0.5] (long dashes), $[1.5,2.5]$ (dashed-dotted). A typical statistical error bar is shown. Results with $r_{0}=2.0 \mathrm{fm}$ (not shown) are close to, and just below, the corresponding points for $r_{0}=1.5 \mathrm{fm}$.

Table 1), showing the effects of transverse hydrodynamic expansion. Moreover, for any given $p_{\perp 0}^{c}$, the width of the distribution of $p_{\perp}^{D}$ (not shown) is consistent with a thermal distribution at $T=150 \mathrm{MeV}$.

In comparing the four data sets in Fig. 1, we find that raising either $r_{0}$ or $d \Gamma / d \ell$ causes a drop in $p_{\perp}^{D}$ (for $p_{\perp 0}^{c}<1700 \mathrm{MeV}$ ). We explain this by selecting two populations of $D$ mesons: those which emerge from flux-tube fission and then escape the system - fragmentation mesons - and those which are trapped within their original droplets until the latter evaporate-breakup mesons. Fig. 2 shows the proportion of breakup mesons for the four data sets. This fraction drops with increasing $p_{\perp 0}^{c}$ and with increasing $d \Gamma / d \ell$, because each of these helps the charmed quark break a flux tube and escape; the fraction also drops with increasing $r_{0}$ because a larger droplet has a longer lifetime and hence affords greater opportunity for escape before breakup. Note that the fraction of breakup mesons lies between $20 \%$ and $75 \%$, so the thermalization of charmed quarks in the plasma is a very important effect.

In Fig. 3 we see that breakup mesons are strongly thermalized, that is, their $p_{\perp}$ is independent of $p_{\perp 0}^{c}$. (Fig. 3 shows results of a simulation with $D \pi$ 


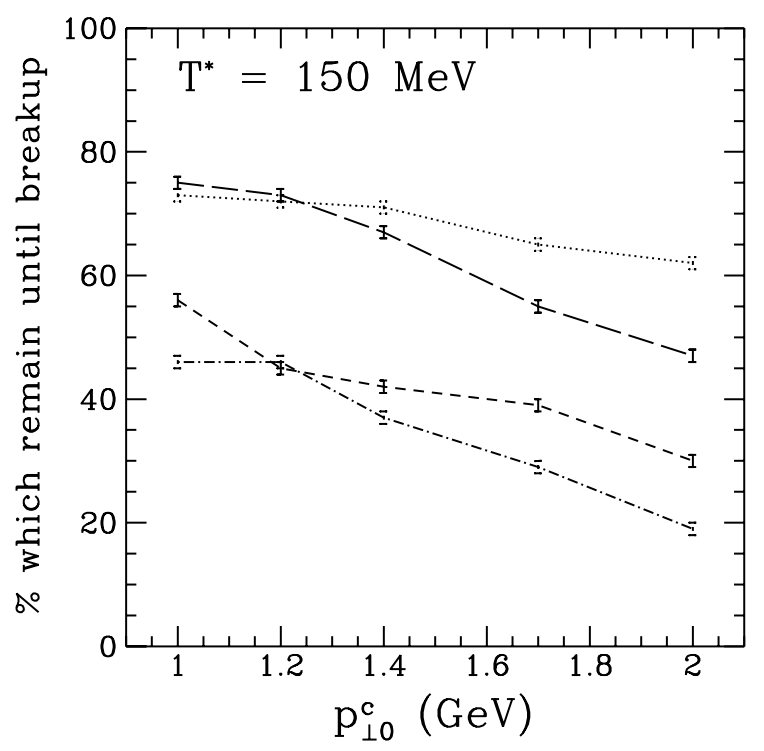

Figure 2: The proportion of $c$ quarks which are trapped inside their original droplets until their breakup, as a function of initial transverse momentum. The four curves are for different values of $r_{0}$ and $d \Gamma / d \ell$ as in Fig. 1.

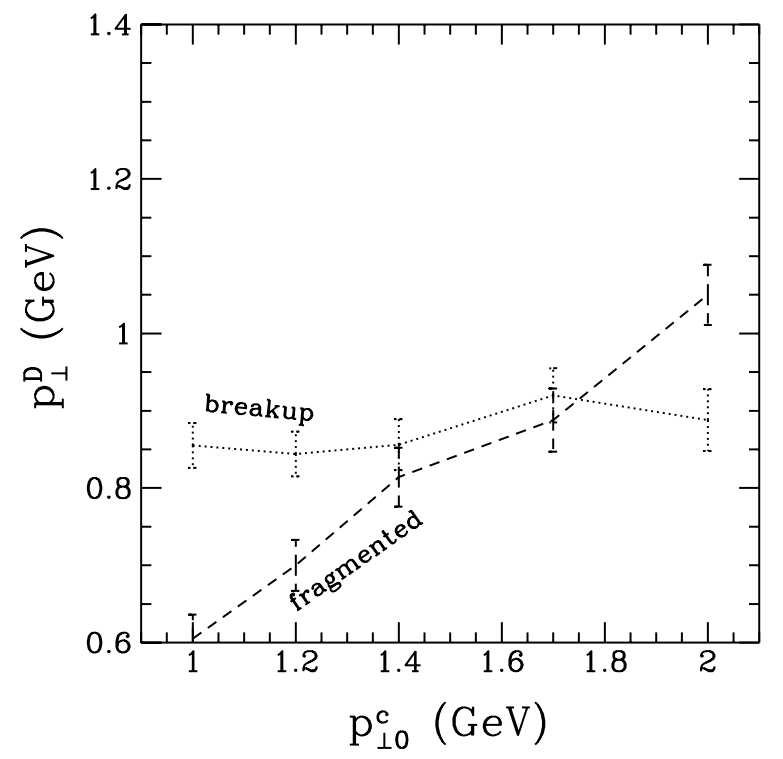

Figure 3: Comparison of two populations of $D$ mesons. The RMS $p_{\perp}$ of $D$ mesons is plotted against the initial $p_{\perp}$ of the $c$ quarks, for breakup mesons (dotted curve) and for fragmentation mesons (dashed curve). Here $T^{*}=150 \mathrm{MeV}, r_{0}=1 \mathrm{fm}, d \Gamma / d \ell=2.5 \mathrm{fm}^{-2}$. 


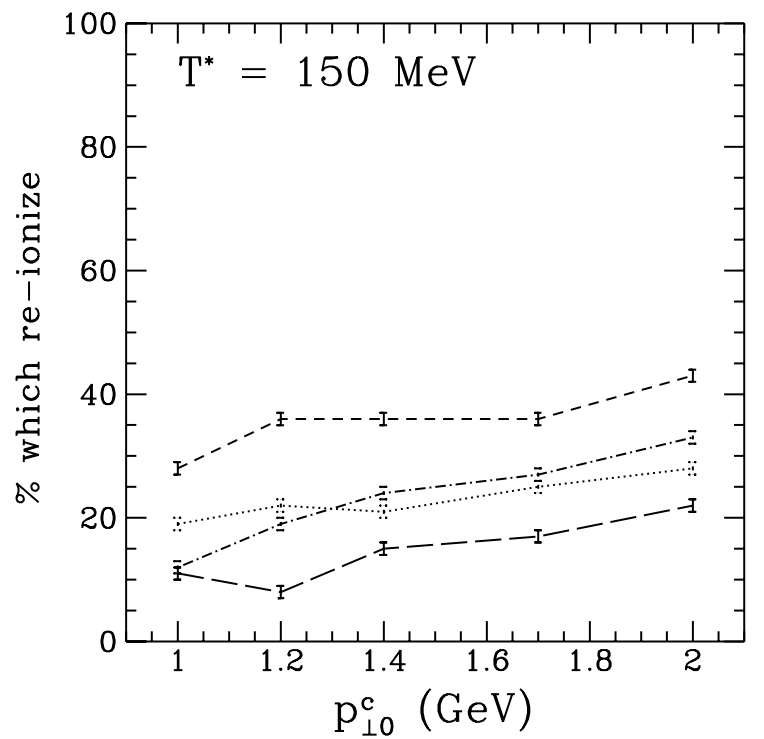

Figure 4: The proportion of $c$ quarks which undergo re-ionization, as a function of initial transverse momentum. The four curves are for different values of $r_{0}$ and $d \Gamma / d \ell$ as in Fig. 11.

scattering turned off, so that hydrodynamic effects are weak and $p_{\perp}^{D}$ is close to its thermal value.) The $p_{\perp}$ of the fragmentation mesons, however, does depend on $p_{\perp 0}^{c}$ and for low values of $p_{\perp 0}^{c}$ it in fact lies below the thermal value; this is due to our static model for the droplet wall, which does not include thermal fluctuations in its position and velocity caused by coupling to the plasma inside. Thus for low values of $p_{\perp 0}^{c}$ the proportion of fragmentation mesons affects $p_{\perp}^{D}$ strongly. Having noted the dependence of this proportion on the parameters $r_{0}$ and $d \Gamma / d \ell$, we now understand the trends in Fig. $\mathbb{1}$.

These trends are strengthened by the phenomenon of re-ionization, that is, reabsorption by a droplet after initial hadronization. The proportion of quarks which undergo re-ionization is shown in Fig. 4. This process sharply lowers the momentum of $c$ quarks. The lower the quark momentum, the less the chances of fragmentation and the greater the probability that the eventual meson will emerge only at breakup of the droplet. This process thus converts fragmentation mesons into a second generation of breakup mesons. (The latter are not represented in Figs. 2 and 3.) The dependence on the parameters is explained as follows. Raising $d \Gamma / d \ell$ will lead to earlier release of the $D$ meson, which puts it into an environment with higher droplet density, which will increase the chances of recapture; lowering $r_{0}$ will increase the number of droplets as $r_{0}^{-3}$, which again will make re-ionization more likely despite the decreased cross section for capture. 


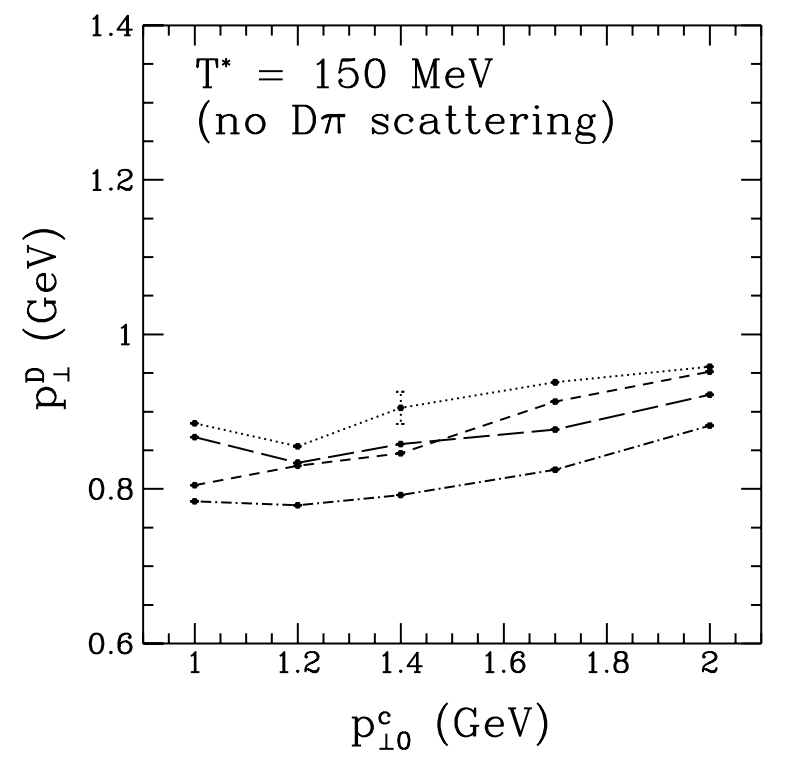

Figure 5: RMS transverse momentum of $D$ meson vs. initial transverse momentum of $c$ quark, with $D \pi$ scattering suppressed. The four curves are for different values of $r_{0}$ and $d \Gamma / d \ell$ as in Fig. 11.

Finally, to show the importance of $D \pi$ scattering, we show results (Fig. 5) of simulations in which it is omitted. Comparison of Fig. 5 with Fig. 1 shows that $D \pi$ scattering causes a systematic increase in $p_{\perp}^{D}$ of about $60 \mathrm{MeV}$. We summarize our findings at $T^{*}=150 \mathrm{MeV}$ as follows: (1) Break-up mesons emerge from the droplets with thermal momenta; (2) fragmentation mesons emerge with lower than thermal momenta; (3) D $\pi$ scattering adds momentum so that the resulting $p_{\perp}^{D}$ is in the neighborhood of its thermal value.

More interesting than this detailed analysis, however, is a comparison to the case of $T^{*}=200 \mathrm{MeV}$, to which we now proceed. Fig. 6 shows that the RMS $p_{\perp}^{D}$ here behaves much the same as for $T^{*}=150 \mathrm{MeV}$, but with a systematic shift upward of $120 \mathrm{MeV}$ for given $r_{0}$ and $d \Gamma / d \ell$. (The thermal value of this quantity is $980 \mathrm{MeV}$, an increase of $160 \mathrm{MeV}$ from $T^{*}=150 \mathrm{MeV}$.) As may be seen in Fig. 7, the bulk of this effect is due to $D \pi$ scattering: Comparison of Fig. 7 with Fig. 5 shows, in the absence of $D \pi$ scattering, almost no difference between the two temperatures. Examination of the counterpart to Fig. 2 shows (see Fig. 8) that the number of breakup mesons has decreased substantially, mainly because thermal motion of $c$ quarks in the droplets is more effective at pushing them over fragmentation thresholds. Having seen that breakup mesons are thermalized while fragmentation mesons are not, we understand why the $D$ mesons, upon emerging from the droplets, are no hotter in spite of the increase in the ambient temperature. It is only the in- 


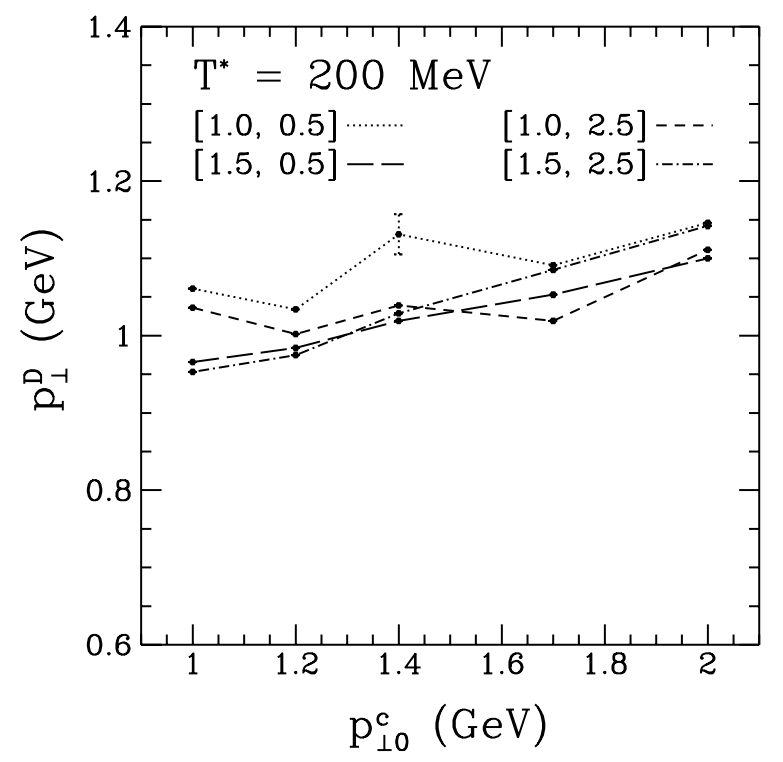

Figure 6: As in Fig. 1, but for $T^{*}=200 \mathrm{MeV}$.

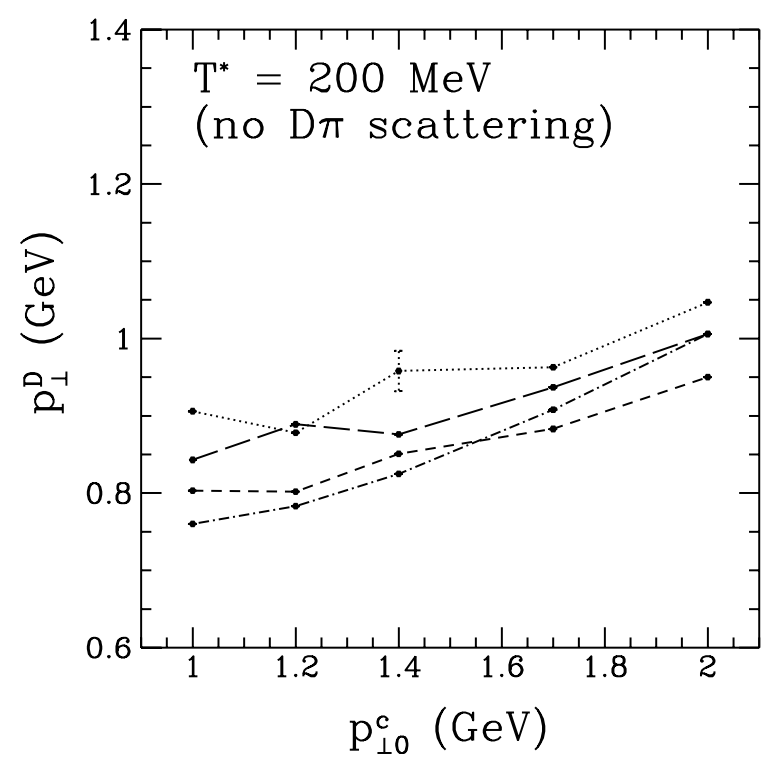

Figure 7: As in Fig. 6 $\left(T^{*}=200 \mathrm{MeV}\right)$, but with $D \pi$ scattering suppressed. 


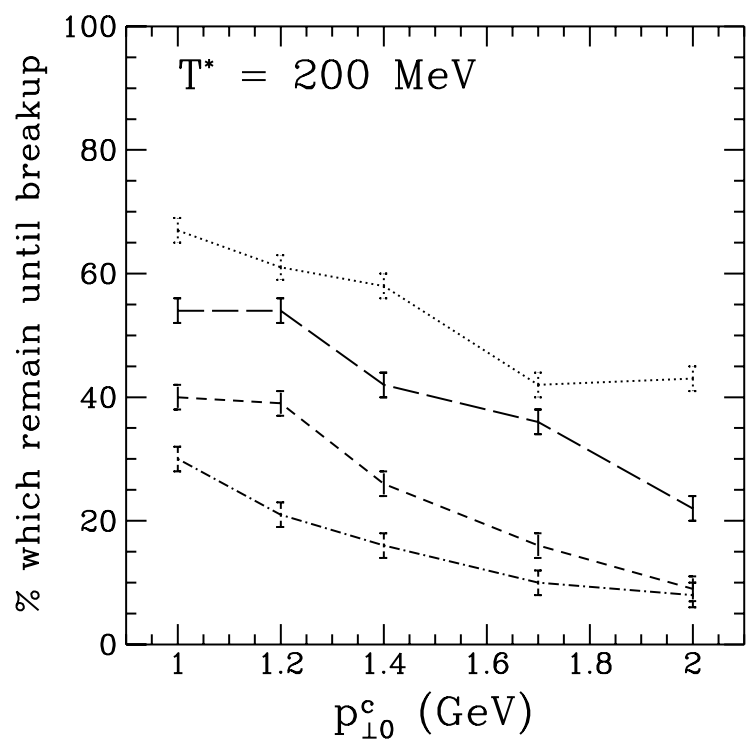

Figure 8: As in Fig. 2, but at $T^{*}=200 \mathrm{MeV}$.

teraction with the hot pion gas that heats the $D$ mesons to thermal momenta and restores their thermometric value.

An experimental measurement of the $p_{\perp}$ distribution of $D$ mesons will reflect a folding of one of the curves in Fig. 1 (or G) with a distribution for $p_{\perp 0}^{c}$. As a source of uncertainty, the weak $p_{\perp 0}^{c}$ dependence is not very troublesome. More significant are the differences among the curves with different parameters $r_{0}$ and $d \Gamma / d \ell$. Nevertheless, as seen in Fig. 9, there is almost no overlap between the range of $p_{\perp}^{D}$ for $T^{*}=150 \mathrm{MeV}$ and that for $T^{*}=200 \mathrm{MeV}$. A measurement of $p_{\perp}^{D}$ will still provide a rough thermometer of the transition temperature, one that can become finer as the assumptions are narrowed down.

Of the parameters we vary, we find that the initial droplet radius affects the $p_{\perp}^{D}$ most strongly; unfortunately, this parameter is the one which is furthest from quantitative understanding. Improvement of our results must therefore await a more detailed model of droplet formation in the phase transition.

\section{Acknowledgements}

We thank S. Gavin, G. Bertsch, and L. McLerran for discussions concerning the cascade hydrodynamics simulation code. This work was supported by a Wolfson Research Award administered by the Israel Academy of Sciences and Humanities, and by the Basic Research Fund of Tel Aviv University. B. 


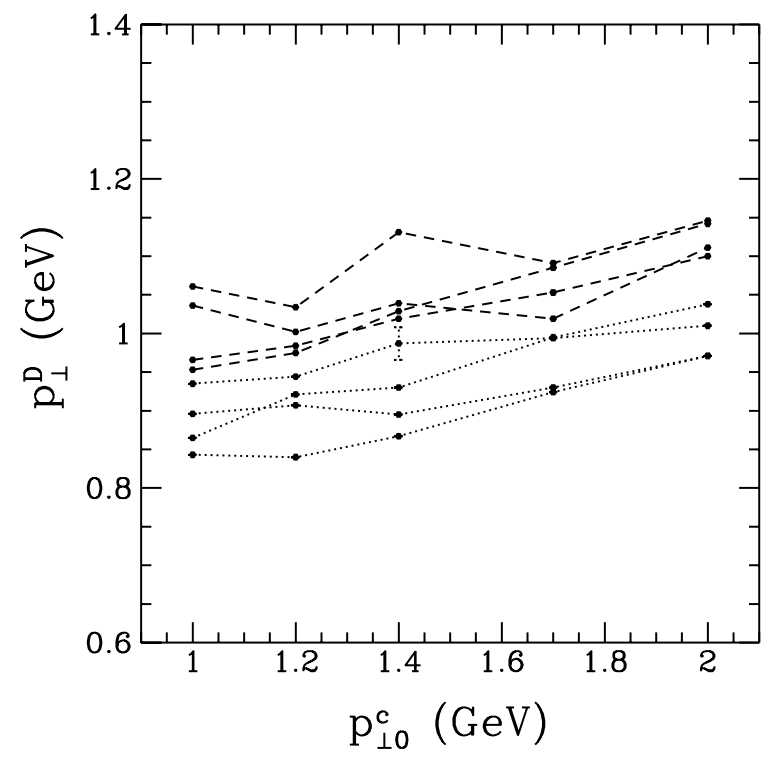

Figure 9: Comparison of results for $T^{*}=150 \mathrm{MeV}$ (dotted curves, same data as in Fig. (1) with results for $T^{*}=200 \mathrm{MeV}$ (dashed curves, same data as in Fig. (6).

S. thanks the European Center for Theoretical Studies in Nuclear Physics and Related Areas (ECT*) in Trento for its hospitality while this paper was being written.

\section{References}

[1] A. Shor, Phys. Lett. 215B, 375 (1988); S. Gavin, P. L. McGaughey, P. V. Ruuskanen, and R. Vogt, LBL preprint LBL-37981 (unpublished, 1996) hep-ph/9604369.

[2] R. V. Gavai, S. Gupta, P. L. McGaughey, E. Quack, P. V. Ruuskanen, R. Vogt, and X.-N. Wang, Int. J. Mod. Phys. A 10, 2999 (1995); I. Sarcevic and P. Valerio, Phys. Rev. C 51, 1433 (1995)

[3] L. D. Landau, Zh. Eksp. Teor. Fiz. 7, 203 (1937), translated in Collected Papers of L. D. Landau, ed. by D. ter Haar (Pergamon, New York, 1965); M. N. Rosenbluth, W. M. MacDonald, and D. L. Judd, Phys. Rev. 107, 1 
(1957); E. M. Lifshitz and L. P. Pitaevskii, Physical Kinetics (Pergamon, New York, 1981), Chap. 2.

[4] B. Svetitsky, Phys. Rev. D 37, 2484 (1988).

[5] D. Levin-Plotnik and B. Svetitsky, Phys. Rev. D 52, 4248 (1995).

[6] J. D. Bjorken, Fermilab preprint PUB-82/59-THY (unpublished, 1982); M. H. Thoma and M. Gyulassy, Nucl. Phys. B351, 491 (1991); E. Braaten and M. H. Thoma, Phys. Rev. D 44, 1298 (1991); ibid. 44, 2625 (1991); Y. Koike and T. Matsui, Phys. Rev. D 45, 3237 (1992)

[7] F. Cooper, G. Frye, and E. Schonberg, Phys. Rev. D 11, 192 (1975).

[8] J. D. Bjorken, Phys. Rev. D 27, 140 (1983).

[9] E. V. Shuryak, Phys. Rev. Lett. 68, 3270 (1992); E. van Doorn, B. Mueller, M. H. Thoma, and X.-N. Wang, Phys. Rev. C 48, 1275 (1993); L. Xiong and E. V. Shuryak, Phys. Rev. C 49, 2203 (1994).

[10] B. Svetitsky, Phys. Lett. 227B, 450 (1989); B. Svetitsky, in The Nuclear Equation of State, ed. by W. Greiner and H. Stöcker (Plenum, New York, 1989), Part B, p. 257.

[11] G. Bertsch, M. Gong, L. McLerran, V. Ruuskanen, and E. Sarkkinen, Phys. Rev. D 37, 1202 (1988).

[12] A. Uziel, M. Sc. thesis, Tel Aviv University (unpublished, 1993, in Hebrew).

[13] T. S. Biro, H. B. Nielsen, and J. Knoll, Nucl. Phys. B 245, 449 (1984).

[14] A. K. Kerman, T. Matsui, and B. Svetitsky, Phys. Rev. Lett. 56, 219 (1986).

[15] H. Satz, Nucl. Phys. A 544, 371c (1992).

[16] R. C. Hwa, Oregon preprint OITS-592 (unpublished, 1995) nuclth/9512002.

[17] V. Weisskopf, Phys. Rev. 52, 295 (1937). 
[18] M. Danos and J. Rafelski, Phys. Rev. D 27, 671 (1983).

[19] B. Banerjee, N. Glendenning, and T. Matsui, Phys. Lett. 127B, 453 (1983).

[20] B. Müller and J. M. Eisenberg, Nucl. Phys. A 435, 791 (1984).

[21] X. Artru and G. Mennessier, Nucl. Phys. B70, 93 (1974); M. G. Bowler, Z. Phys. C11, 169 (1981); B. Andersson, G. Gustafson, and B. Söderberg, Z. Phys. C20, 317 (1983); B. Andersson, G. Gustafson, G. Ingelman, and T. Sjostrand, Phys. Rep. 97, 33 (1983); T. D. Gottschalk, Nucl. Phys. B239, 349 (1984).

[22] M. Binkley et al., Phys. Rev. Lett. 48, 73 (1982).

[23] J. Busetnitz et al., Phys. Rev. D 40, 1 (1989).

[24] P. Estabrooks et al., Nucl. Phys. B95, 30 (1977). 